\title{
Full-field optical measurement of curvatures in ultra-thin-film-substrate systems in the range of geometrically nonlinear deformations
}

\author{
Hansuk Lee and Ares J. Rosakis ${ }^{a}$ \\ Graduate Aeronautical Laboratories, California Institute of Technology, Mail Code 105-50, Pasadena, \\ California 91125 \\ L. B. Freund ${ }^{\text {b) }}$ \\ Division of Engineering, Brown University, 182 Hope Street, Providence, Rhode Island 02912-9104
}

(Received 2 October 2000; accepted for publication 19 February 2001)

\begin{abstract}
This article describes coherent gradient sensing (CGS) as an optical, full-field, real-time, nonintrusive, and noncontact technique for the measurement of curvatures and nonuniform curvature changes in film-substrate systems. The technique is applied to the study of curvature fields in thin $\mathrm{Al}$ films $(6 \mu \mathrm{m})$ deposited on thin circular silicon wafers $(105 \mu \mathrm{m})$ of "large" in-plane dimensions (50.8 $\mathrm{mm}$ in diameter) subjected to thermal loading histories. The loading and geometry is such that the system experiences deformations that are clearly within the nonlinear range. The discussion is focused on investigating the limits of the range of the linear relationship between the thermally induced mismatch strain and the substrate curvature, on the degree to which the substrate curvature becomes spatially nonuniform in the range of geometrically nonlinear deformation, and finally, on the bifurcation of deformation mode from axial symmetry to asymmetry with increasing mismatch strain. Results obtained on the basis of both simple models and more-detailed finite-element simulations are compared with the full-field CGS measurements with the purpose of validating the analytical and numerical models. (C) 2001 American Institute of Physics.
\end{abstract}

[DOI: $10.1063 / 1.1364650]$

\section{INTRODUCTION}

A material configuration of central importance in microelectronics, optoelectronics, and thermal barrier coating technology, among other areas of application, is a thin film of one material deposited onto a substrate of another material. Fabrication of such a structure inevitably gives rise to stress in the film due to lattice mismatch, differing coefficients of thermal expansion, chemical reactions, or other physical effects. Experimental techniques for stress measurement in thin films, which are based on the observation of substrate curvature induced by this stress, are gaining increasingly widespread use as diagnostic procedures. Applications for metal films on semiconductor substrates are discussed by Flinn ${ }^{1}$ and $\mathrm{Nix}^{2}$, and recent refinements for in situ measurements of curvature during vapor deposition are described by Floro and co-workers. ${ }^{3-5}$

Most data on substrate curvature are interpreted on the basis of the classical Stoney formula. ${ }^{6}$ In its most basic form, this formula provides an expression for the curvature $\kappa_{\mathrm{St}}$ of the substrate in terms of the film membrane force $f$ (force per unit distance) due to stress as

$$
\kappa_{\mathrm{St}}=\frac{6 f}{h_{s}^{2} M_{s}},
$$

where $h_{s}$ is the substrate thickness, $M_{s}$ is its biaxial elastic modulus, and $f$ is the membrane tension in the film. Stated in this way, formula (1) involves the elastic properties of the

\footnotetext{
a)Electronic mail: rosakis@atlantis.caltech.edu

${ }^{\mathrm{b})}$ Electronic mail: freund@engin.brown.edu
}

substrate, the substrate thickness $h_{s}$, and the through-thethickness variation of mismatch strain only through $f$. Formula (1) follows from analysis of a film-substrate system which is based on several linearity assumptions. ${ }^{7-10}$ Current measurement technology is being applied to ever-thinner substrates in order to extend the sensitivity range of the measurements, particularly in research on fundamental stress issues during deposition. For example, in situ measurements of stress during epitaxial growth of a silicon-germanium alloy onto silicon crystals, substrates only a few hundred microns thick with lateral extent of a few centimeters, are currently being used. The aspect ratios (lateral dimension/thickness) of such substrates fall within the range of 50-1000, so they are "thin structures" indeed. A consequence is that states of deformation are readily achieved for which nonlinear geometrical effects come into play. Thus, the purpose here is to discuss aspects of curvature due to a bonded, stressed thin film which are relevant to the interpretation of substrate curvature experiments. The discussion focuses first on axially symmetric, nonlinear deformations, and then on deformations which may be asymmetric. To make the discussion definite, attention will be restricted to a system with a circular substrate of radius $R$, the only lateral dimension of consequence. It will be assumed that the film material is also homogeneous and the stress in the film is uniform throughout. Arbitrary through the thickness variation of properties or mismatch strain was considered by Freund ${ }^{7}$ and by Finot and Suresh. ${ }^{10}$

A polar section of the system is shown in Fig. 1. The elastic biaxial modulus and Poisson ratio of the film are $M_{f}, \nu_{f}$ and similarly for the substrate. Cylindrical $(r, \theta, z)$ 


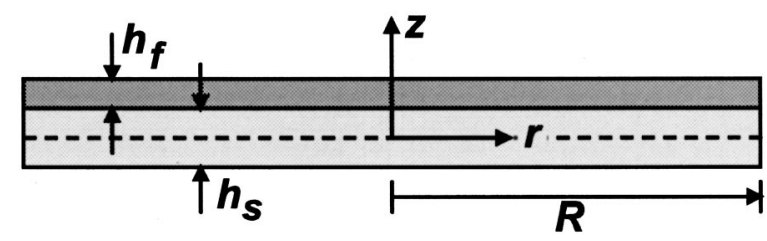

FIG. 1. Axial section of the film-substrate system showing the polar coordinates and the physical dimensions: film thickness $h_{f}$, substrate thickness $h_{s}$, and substrate radius $R$.

coordinates are adopted with the origin on the geometrical midplane of the substrate. The mismatch strain, which is the stress-free strain that would render the film compatible with the substrate, is denoted by $\varepsilon_{m}$. The associated mismatch stress is $\sigma_{m}=M_{f} \varepsilon_{m}$ and the membrane force $f=M_{f} \varepsilon_{m} h_{f}$ in this case.

Although thin-film behavior has emerged as a research area in mechanics only recently, the general issue of large deformation of thin plates has been of interest for many years. In addition to those references cited above, Friedrichs and Stoker ${ }^{11,12}$ studied the axially symmetric postbuckling behavior of a thin, circular plate subjected to uniform inplane compression along its edge; Wittrick ${ }^{13}$ considered the axially symmetric snap-through buckling of a bimetallic disk under temperature change; and Hyer ${ }^{14}$ described the warping of layered composite materials due to residual stress. In this article, we first summarize the results of a recent analysis by Freund, ${ }^{15}$ who modeled the complex nonlinear deformation behavior of thin-film/substrate structures. We then compare the analytical predictions with real-time experimental measurements. It should be noted at this point that nonlinear distortions of large wafers were investigated experimentally by Finot et al., ${ }^{16}$ who used a geometrical grid projection method to record curvatures resulting from a variety of deposition conditions and geometrical parameters.

Techniques based on optical interferometry offer much promise as a means for real-time, remote, high-resolution, full-field measurements of curvature and curvature changes. However, standard interferometric techniques (e.g., Twyman-Green interferometer) are sensitive to rigid-body rotation and displacement of the specimen surface, and thus are very vibration sensitive. Moreover, since these interferometric techniques measure the surface topography, two successive differentiations of the experimental data are required to obtain curvature. This often results in error levels that are unacceptable, and drastically reduces the potential of such methods for accurate stress measurement in thin-film structures. Rosakis et al. ${ }^{17}$ have employed the optical technique of coherent gradient sensing (CGS) to measure the entire curvature tensor fields in thin-film and micromechanical structures. The CGS technique offers significant advantages over other currently used curvature measurement techniques. These advantages include rigid-body motion insensitivity, and the associated vibration insensitivity and accurate and full-field measurement of all components of the curvature tensor as well as in situ and real-time capabilities. In the second part of this study, we use CGS to record the evolution of curvature fields in the nonlinear deformation regime of thin-film/substrate structures subjected to thermal loading. The experimental measurements provide bench-mark comparisons for the validation of the assumptions involved in Freund's ${ }^{15}$ nonlinear analysis and of finite-element models used in the current investigation.

\section{AXIALLY SYMMETRIC DEFORMATION}

For axially symmetric deformation of the substrate, the only nonzero stress components in polar coordinates are $\sigma_{r r}(r, z)$ and $\sigma_{\theta \theta}(r, z)$. For the case of thin plate-like configurations (see Fig. 1), the Kirchhoff hypothesis can be adopted, which implies that material lines initially normal to the substrate midplane remain so during deformation. The strain components can then be written in terms of the displacement components $u_{r}(r), u_{z}(r)$ of the substrate midplane as

$$
\begin{aligned}
& \varepsilon_{r r}(r, z)=u_{r}^{\prime}(r)+\frac{u_{z}^{\prime}(r)^{2}}{2}-z u_{z}^{\prime \prime}(r)+\varepsilon_{m}, \\
& \varepsilon_{\theta \theta}=\frac{u_{r}(r)}{r}-z \frac{u_{z}^{\prime}(r)}{r}+\varepsilon_{m},
\end{aligned}
$$

where the prime denotes differentiation with respect to $r$. The definition of mismatch strain is extended so that $\varepsilon_{m}$ $\equiv 0$ in the substrate; in this case, Eq. (2) applies for both materials. Expressions (2) are the usual linear-straindisplacement relations for axially symmetric deformation of a circular plate, augmented by the term $u_{z}^{\prime}(r)^{2} / 2$ to account for midplane stretching due to finite rotation of the substrate normal about an axis in the $\theta$ direction. This nonlinear aspect of the strain-displacement relations is the essential feature of the von Karman elastic-plate theory.

As a first calculation, parametric forms of $u_{r}(r), u_{z}(r)$ are adopted. The principle of stationary potential energy is then invoked to determine optimal values of the parameters involved. Consider the particular choice $u_{r}=\varepsilon_{0} r+\varepsilon_{1} r^{3}, u_{z}$ $=\kappa r^{2} / 2$, which preserves the constant spherical curvature feature of the small deflection analysis as an assumption in the nonlinear range. The total potential energy is then a function of the parameters $\varepsilon_{0}, \varepsilon_{1}$, and $\kappa$. Following Freund, ${ }^{15}$ the requirement that it be stationary with respect to these variables leads to the relationship

$$
S=K\left\lfloor 1+\left(1-\nu_{s}\right) K^{2}\right\rfloor,
$$

for the case when $h_{f} \ll h_{s}$, where $S=3 \varepsilon_{m} R^{2} h_{f} M_{f} / 2 h_{s}^{3} M_{s}$ is the normalized mismatch strain and $K=R^{2} \kappa / 4 h_{s}$ is the normalized curvature. This result is shown graphically in Fig. 2, along with the corresponding linear relationship (Stoney formula) between $K$ and $S$. This result implies that the system parameters for which $S>0.3$ result in a response in the range of nonlinear geometrical effects. Quantitative conclusions regarding the behavior within the nonlinear range cannot be drawn at this point because of the uncertainty of the severe constraint implied by the assumption of spatially uniform curvature. This constraint is relaxed in the numerical analysis discussed below.

For the case when $h_{f} \ll h_{s}$, and when the film strain is not changed appreciably as a result of substrate deformation, 


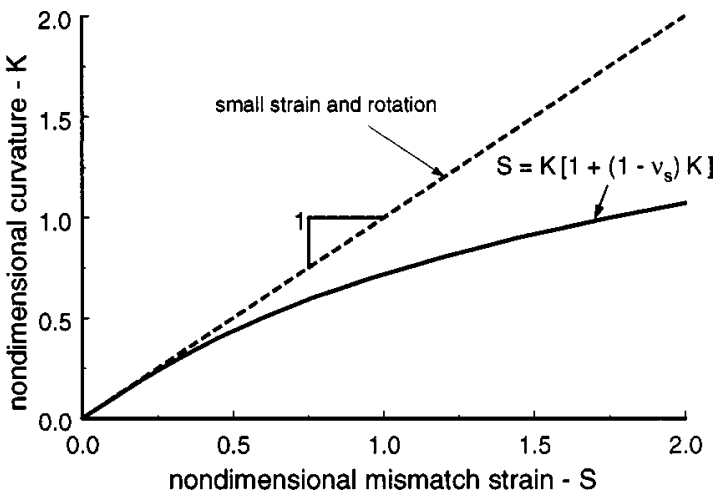

FIG. 2. Graph of the relationship between normalized curvature $K$ and normalized mismatch strain $S$ implied by Eq. (3) with $\nu=1 / 4$ for large deflection with spatially uniform substrate curvature. The dashed line represents the linear relationship implied by small deflection theory.

the equilibrium equations in terms of midplane displacements have been studied by Friedrichs and Stoker ${ }^{12}$ in the context of postbuckling behavior of circular plates. Some useful observations follow from examination of these nonlinear equations. For example, suppose the solution for the displacements $u_{r}(r), u_{z}(r)$ is known for some values of the system parameters $\varepsilon_{m}, M_{s}$, and $M_{f}$ and for some dimensions $R, h_{f}$, and $h_{s}$. If all dimensions are then scaled by a positive factor, say $\alpha$, holding the other system parameters fixed, the displacements for the scaled system are $\alpha u_{r}(r)$ and $\alpha u_{z}(r)$. Thus, the solutions of the nonlinear equations scale with the absolute size of the structure, so only one size need be considered for a complete description (at a given aspect ratio). Consequently, all lengths can be normalized with respect to substrate radius $R$.

A second observation is that the parameters $\varepsilon_{m}, M_{f}$, and $h_{f}$ enter only through the combination $\varepsilon_{m} M_{f} h_{f}$, which is the membrane force $f$ in the film. All situations with a certain value of $f$ are identical at this level of approximation, no matter what values the individual parameters $\varepsilon_{m}, M_{f}$, and $h_{f}$ may assume. For this reason, the dimensionless parameter $S$, which was introduced above and which involves these parameters in the same combination, will be adopted to represent the magnitude of mismatch strain in the subsequent discussion.

The deformation is analyzed in greater detail by means of the Abaqus finite-element code. A result in the form of level curves of curvature $K$ in the $(r / R, S)$ plane is shown in Fig. 3 for the case when $h_{s} / h_{f}=20$ and $R / h_{s}=50$. The curvature is essentially constant over the entire substrate at a given level of mismatch strain as long as the level curves of curvature are parallel to the radial distance axis for $0 \leqslant r / R$ $\leqslant 1$. This is indeed the case for normalized curvature varying between zero and a value of about 0.3 . Because of the normalization convention for curvature and mismatch strain adopted here, this implies that the corresponding normalized mismatch strain is also in the range $0 \leqslant S \leqslant 0.3$. Thus, the implied limit on the range of linear response found here is consistent with the result represented in Fig. 2. Similar conclusions were reported by Finot and Suresh ${ }^{16}$ on the basis of nonlinear finite-element calculations.

As the normalized mismatch strain $S$ is increased above

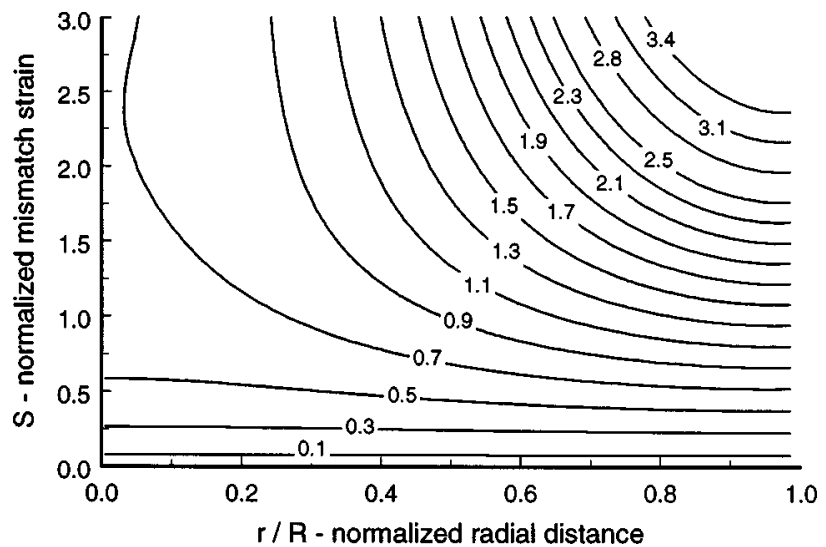

FIG. 3. Contour plot showing level curves of normalized curvature $K(r)$ for axially symmetric deformation in the plane with normalized distance $r / R$ as coordinate on the horizontal axis and normalized mismatch strain $S$ on the vertical axis for common Poisson ratio $\nu=1 / 4$. Uniform curvature requires the level curves to be parallel to the horizontal axis.

the value 0.3 , the curvature distribution becomes increasingly nonuniform. The general trend is that the curvature assumes values substantially below the average curvature for portions of the substrate near its center, and it takes on values substantially above the average value near the periphery of the substrate. For example, for a normalized mismatch strain of $S=2$, the normalized curvature varies from about $K(0)$ $\approx 0.55$ at the substrate center to a value of about $K(R)$ $\approx 1.3$ at the substrate edge.

The feature that level curves of curvature in Fig. 3 eventually assume a tangent direction which is parallel to the strain axis near $r / R=0$ implies that the curvature near the center of the wafer first increases as the spatially uniform strain $S$ increases. Eventually, the curvature reaches a maximum value at some level of $S$, and then decreases with further increase in strain beyond this level. This behavior is a direct consequence of the nonlinearity of the deformation.

\section{BIFURCATION OF EQUILIBRIUM SHAPES}

Imagine the mismatch strain in the film being increased in magnitude from the zero initial value. For relatively small values, the deformed shape of the substrate is essentially spherical as long as the response remains within the linear range. Once $\varepsilon_{m}$ becomes large enough in magnitude to bring the system into the range of a geometrically nonlinear response, the deformed shape may continue to be axially symmetric. However, this deformation mode requires that the substrate must deform in extension as well as in bending, and the stiffness against such deformation is very large compared to the bending stiffness at comparable levels of surface strain. On the other hand, cylindrical bending, or generalized plane strain bending, can occur with no midplane extension. This suggests that, at some point as the magnitude of $\varepsilon_{m}$ increases, the system may begin a transition from axially symmetric deformation toward cylindrical bending deformation. Such a transition would represent a bifurcation of equilibrium states.

Before proceeding with a full numerical simulation, a simple variational approach is taken. The transverse deflec- 
tion of the substrate midplane is assumed to be $u_{z}(x, y)$ $=\left(\kappa_{x} x^{2}+\kappa_{y} y^{2}\right) / 2$, where $\kappa_{x}$ and $\kappa_{y}$ are the (spatially uniform) principal curvatures of the deformed shape. A strain distribution consistent with this deflection, which accounts for midplane stretching, is also assumed..$^{15}$ The principle of stationary potential energy is then invoked to determine the relationship of the principal curvatures to the system parameters, which is necessary for the system to be in equilibrium. This development follows the work of Masters and Salamon ${ }^{18}$ and Salamon and Masters. ${ }^{19}$ A more-detailed examination of bifurcation on the basis of a finite-element simulation without a priori restrictions on the deformation beyond the Kirchhoff hypothesis is then described.

The goal in this development is to extract fairly simple analytical results, which are merely representative of the phenomena of interest. As before, attention is restricted to systems for which the elastic response of both the film and substrate materials is isotropic, and the moduli of the film and substrate are the same. While it is not necessary to place restrictions on the relative thickness $h_{f} / h_{s}$ for this development, results are presented under the assumption that $h_{f} / h_{s}$ $\ll 1$ in order to be consistent with other parts of the discussion.

For the present case, the equilibrium conditions can be obtained in closed form. ${ }^{18,20}$ In particular, for a given mismatch strain and geometric parameters, a relationship between $\kappa_{x}$ and $\kappa_{y}$ is obtained, which represents the locus of equilibrium states for the system in the plane of $\kappa_{x}$ vs $\kappa_{y}$, namely,

$$
\left(\kappa_{x}-\kappa_{y}\right)\left\lfloor\kappa_{x} \kappa_{y} R^{4}(1+\nu)-16\left(h_{s}+h_{f}\right)^{2}\right\rfloor=0,
$$

where $\nu$ is the common Poisson ratio. This result is exact within the class of deformations under consideration. An important consequence of admitting the possibility of finite deflections is evident in Eq. (4). It is clear that a spherical deformed shape of the system, with $\kappa_{x}=\kappa_{y}$, is still an equilibrium shape (although the magnitude of the spherical curvature differs significantly from that predicted on the basis of the small deflection theory for a given mismatch strain, as was noted in the preceding section). The new feature is the possibility of a second asymmetric equilibrium shape represented by the vanishing of the term in square brackets in Eq. (4).

The locus of possible equilibrium curvatures is plotted in Fig. 4 (solid curves) for $\nu=1 / 4$. The straight line bisecting the quadrant represents the spherical shape with $\kappa_{x}=\kappa_{y}$. The curved branch of the locus is obtained by setting the second factor in Eq. (4) equal to zero, and it represents asymmetric deformation, that is, $\kappa_{x} \neq \kappa_{y}$. The intersection point of these two branches is a bifurcation point. For values of spherical curvature on the branch with $\kappa_{x}=\kappa_{y}$, which are less than the curvature at the bifurcation point, it is found that the equilibrium value of potential energy is a local minimum under variations in curvature. Thus, that part of the symmetric branch represents stable equilibrium configurations. On the other hand, for values of spherical curvature which are larger than the curvature at bifurcation, the stationary value of potential energy is found to be a saddle point, so that part of the symmetric branch represents unstable equi-

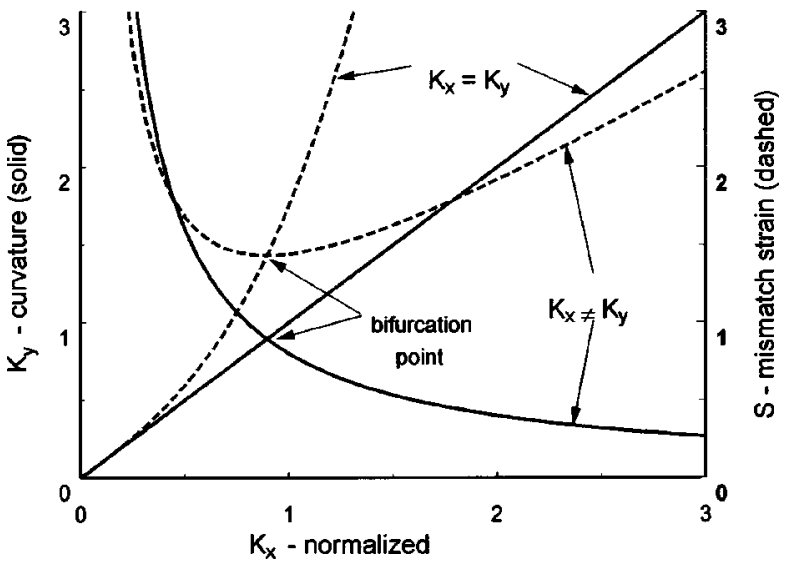

FIG. 4. Relationship between normalized principal curvatures $K_{x}$ and $K_{y}$ implied by equilibrium condition (4) for $\nu=1 / 4$. The intersection between the branch $K_{x}=K_{y}$ corresponding to spherical curvature and the hyperbolic branch is the bifurcation point.

librium configurations. All equilibrium configurations on the asymmetric branch in Fig. 4 (except that corresponding to the bifurcation point itself) are found to be stable configurations.

If the strain magnitude is increased beyond the value corresponding to the bifurcation point, then the deformation becomes asymmetric with the curvature increasing in one direction and decreasing in the orthogonal direction. The principal directions of the asymmetric deformation are completely arbitrary, of course. The bifurcation is stable, in the sense that an increasing strain is required to move the equilibrium configuration away from the bifurcation point along the asymmetric branch in Fig. 4. As the strain magnitude increases further, the equilibrium shapes become more and more asymmetric, approaching a cylindrical-limiting shape with $\kappa_{x} / \kappa_{y} \rightarrow 0$ or $\rightarrow \infty$.

The variation of mismatch strain $\varepsilon_{m}$ along the equilibrium paths corresponding to the equilibrium configurations is also illustrated in Fig. 4 (dashed curves). Again, the mismatch strain is normalized in such a way that the graph is universal, for the choice of $\nu=1 / 4$. The nonlinear relationship between curvature and mismatch strain prior to bifurcation is identical to that shown in Fig. 2.

The results in Fig. 4 are valid for the full range of geometrical parameters for systems which meet the general characteristics of compliant free-standing layers. To give an impression of the magnitudes of parameters involved, consider the state represented by the bifurcation point in Fig. 4 . If the geometry of the system is characterized by $h_{s} / R$, the ratio of substrate thickness to radius, and by $h_{f} / h_{s}$, the ratio of film thickness to substrate thickness, then the normalized spherical curvature and mismatch strain at the bifurcation point are given by

$$
K_{(\mathrm{bif})}=\frac{1}{\sqrt{1+\nu}}, \quad S_{(\mathrm{bif})}=\frac{2}{(1+\nu)^{3 / 2}} .
$$

For $\nu=1 / 4$, the value of $S_{(\text {bif) }}$ is about 1.43 .

The foregoing bifurcation analysis is based on the assumption of spherical curvature of the substrate midplane 


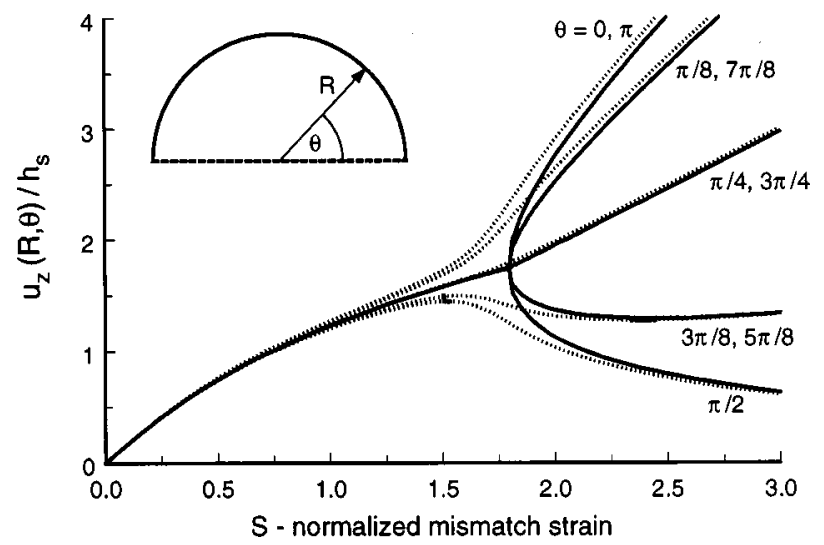

FIG. 5. Plots of normalized transverse deflection $u_{z}(R, \theta) / h_{s}$ at nine points around the circumference of the substrate midplane vs normalized mismatch strain $S$ for $\nu=1 / 4$. These results were obtained by finite-element calculation, and they involve no a priori assumptions on the deformed shape. The dotted curves represent the equivalent result with a $\pm 1 \%$ anisotropy in the mismatch strain.

prior to bifurcation and on the assumed transverse deflection with spatially uniform principal curvatures following bifurcation. Therefore, the bifurcation analysis is repeated without a priori assumptions on the deformed shape of the substrate midplane by means of the numerical finite-element method. The calculations were carried out under the assumption that the deformation has at least one plane of reflective symmetry. The midplane of the $180^{\circ}$ sector of the substrate was covered with a regular radial-circumferential mesh. An eight-noded plate element was prescribed in each mesh segment. The element adopted admits through-the-thickness variation of material properties, and the film-substrate system was defined by prescribing the appropriate variation. Mismatch strain was imposed by specifying a coefficient of thermal expansion for the film material relative to the substrate material, and by making temperature the imposed loading parameter. The temperature was gradually increased from the zero-initial value, and the equilibrium shape for large deflections was computed.

To precipitate stable deformation beyond the point of bifurcation, a slight imperfection in the system was introduced in the form of an anisotropic mismatch strain. Typically, the mismatch strain in the $x$ direction ( $y$ direction) was taken to be $0.01 \%$ larger (smaller) than the value of the nominal $\varepsilon_{m}$. With this level of imperfection, the deformation prior to bifurcation was essentially indistinguishable from the results based on an a priori assumption of axial symmetry, the bifurcation point was sharply defined in each case and reproducible from case to case, and the postbifurcation behavior was stable and reproducible.

The general response observed as the mismatch strain was increased was a range of axially symmetric deformation, with the substrate midplane curvature becoming ever more nonuniform. Then, over a very narrow range of values of nominal mismatch strain, the midplane showed first a slight waviness in the circumferential direction (compared to the substrate thickness) followed by large amplitude waviness in the circumferential direction. This behavior is illustrated in Fig. 5, which shows plots of the transverse deflection nor- malized by substrate thickness $h_{s}$ at nine points around the outer edge of the substrate versus normalized mismatch strain $S$. As $S$ increases from zero, all nine points at first experience the same transverse deflection within the resolution of a graph. As $S$ increases through the value of about 1.77, a fairly sharp transition in response occurs. The deflections at the points on $r=R$ at $\theta=0$ and $\theta=\pi$ increase dramatically for a very small increase in $S$, while the deflection at the point on $\theta=\pi / 2$ decreases dramatically. The deflections at the points on $r=R$ at $\theta=\pi / 4$ and $3 \pi / 4$, on the other hand, remain nearly unchanged.

It is noted that the behavior of thin structures of the kind being discussed here is strongly sensitive to imperfections in the system. To illustrate the point in the present context, the calculation which led to the solid curves in Fig. 5 was redone with a mismatch strain that is $1 \%$ larger (smaller) than the nominal value $\varepsilon_{m}$ in the $x$ direction ( $y$ direction). The result is shown by dotted curves in Fig. 5, where it can be seen that a $1 \%$ imperfection in mismatch strain obliterates the sharp bifurcation transition. Instead, the system undergoes a long, gradual transition from axially symmetric deformation to asymmetric deformation as $S$ increases. There are other significant features of the behavior illustrated in Fig. 5. Among these are (i) the axially symmetric response is nonlinear for values of $S$ beyond about 0.3 , consistent with the behavior observed in Fig. 3; (ii) the maximum deflection at the substrate periphery reaches a value of about two times the substrate thickness before bifurcation occurs; and (iii) the postbifurcation deformation becomes more like cylindrical bending as $S$ increases further beyond 1.77 .

Another noteworthy aspect of the result illustrated in Fig. 5 is the apparent insensitivity of the bifurcation results to variations in the aspect ratio of the substrate. Calculations were carried out for $R / h_{s}=50,100$, and 200. The plots for the three cases, when expressed in terms of the normalized parameters adopted in Fig. 5, are virtually indistinguishable. The behavior of a given system at a fixed level of mismatch strain $\varepsilon_{m}$ will indeed depend on the aspect ratio $R / h_{s}$. However, with the rather natural normalization embodied in the definition of $S$, the horizontal scale in Fig. 5 can be viewed as strain at fixed aspect ratio (as is done here) or, equivalently, as aspect ratio at fixed strain. In other words, the sensitivity of behavior to both mismatch strain and aspect ratio is captured by the single parameter $S$. Note that the ratios $h_{s} / h_{f}=20$ and $M_{s} / M_{f}=1$ were maintained in all calculations so that the film is always relatively thin and the effects of modulus difference are not considered.

\section{FULL-FIELD CURVATURE MEASUREMENT USING COHERENT GRADIENT SENSING}

Figure 6 shows a schematic of the CGS setup in reflection. A coherent, collimated laser beam is directed to the specularly reflecting specimen surface by means of a beam splitter. The reflected beam from the specimen then passes through the beam splitter and is then incident upon a pair of identical high-density (40 lines $/ \mathrm{mm}$ ) Ronchi gratings, $G_{1}$ and $G_{2}$, separated by a distance $\Delta$. The diffracted orders from the two grating are spatially filtered using a filtering lens to form distinct diffraction spots on the filter plane. An aperture 


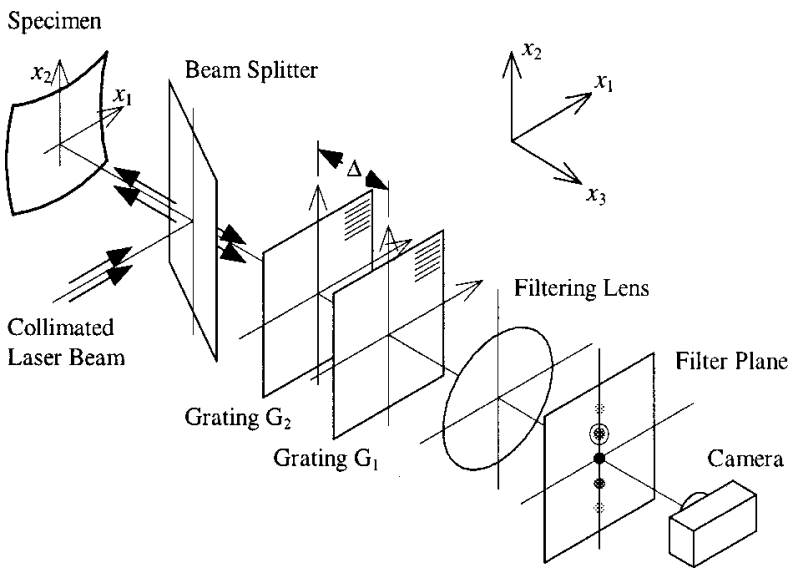

FIG. 6. Schematic of the CGS setup in reflection mode.

placed in this plane serves to filter out the diffraction order of interest, which is then imaged onto the film plane. For our purpose, either of the \pm 1 diffraction orders is of interest, as will become clear in the following discussion.

Figure 7 illustrates the working principle of CGS in two dimensions. Consider an optical wave front incident on the grating pair and let both the gratings have their rulings along the $x_{2}$ axis. A wave front incident on the primary grating, $G_{1}$, is diffracted into several wave fronts denoted as $E_{0}, E_{1}, E_{-1}, E_{2}, E_{-2}$, etc. For illustrative purposes, only $E_{0}, E_{1}$, and $E_{-1}$ are shown in Fig. 7. Each of these wave fronts is further diffracted by the second grating, $G_{2}$, to give rise to wave fronts denoted as $E_{0,0}, E_{0,1}$, $E_{0,-1}, \ldots, E_{1,0}, E_{1,1}, E_{1,-1}, \ldots, E_{-1,0}, E_{-1,1}, E_{-1,-1}, \quad$ etc. Again, only some of the diffracted wave fronts are shown. Now, various sets of parallel diffracted beams are combined using the filtering lens to form diffraction spots $D_{+1}, D_{0}, D_{-1}, \ldots$, in the filter plane (which coincides with the focal plane of the lens). For example, $E_{0,1}$ and $E_{1,0}$ interfere to give diffraction spot $D_{+1} ; E_{1,-1}, E_{0,0}$, and $E_{-1,1}$ interfere to give $D_{0}$, etc. An aperture is placed on the filter plane to block all but the $D_{+1}$ diffraction spot. Subsequently, this diffraction spot is imaged onto the film plane.

Assume that the optical wave front incident on the first grating, $G_{1}$, is approximately planar and has a local phase difference given as $S\left(x_{1}, x_{2}\right)$. The net effect of the two gratings is to produce a lateral shift, or "shearing," of the incident wave front. Thus, the optical wave front along the diffracted beam $E_{1,0}$ [given by $S\left(x_{1}, x_{2}+\omega\right)$ ] is shifted by an

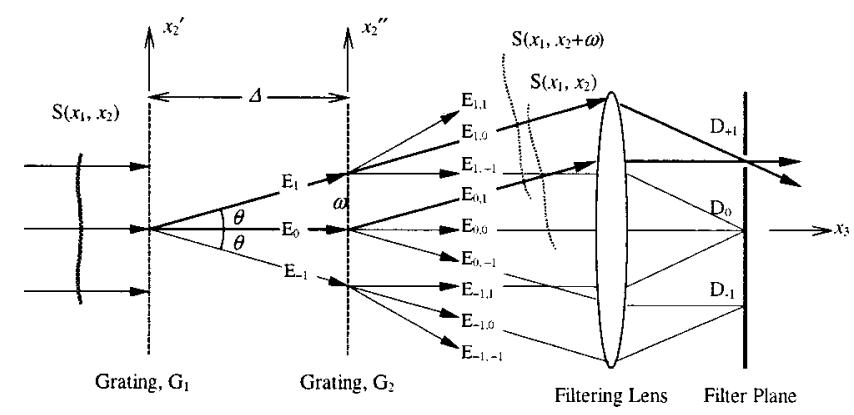

FIG. 7. Schematic illustrating the working principle of CGS. amount $\omega$ along the $x_{2}$ direction as compared to the wave front along the diffracted beam $E_{0,1}$ [given by $S\left(x_{1}, x_{2}\right)$ ]. The wave-front shift is parallel to the principal axis of the gratings, i.e., along $x_{2}$ if the grating lines are oriented along $x_{1}$, as shown in Fig. 7. Moreover, the magnitude of the shift is a function of the grating separation $\Delta$ and the diffraction angle $\theta$ as

$$
\omega=\Delta \tan \theta,
$$

where the diffraction angle $\theta$ is given by

$$
\theta=\arcsin \frac{\lambda}{p},
$$

with $\lambda$ the wavelength of light and $p$ being the grating pitch. For a small angle of diffraction, Eqs. (6) and (7) approximate to

$$
\begin{aligned}
& \omega \approx \Delta \theta, \\
& \theta \approx \frac{\lambda}{p} .
\end{aligned}
$$

Now, consider the interference of the original and shifted wave fronts. The conditions for constructive interference may be expressed as

$$
S\left(x_{1}, x_{2}+\omega\right)-S\left(x_{1}, x_{2}\right)=n^{(2)} \lambda, \quad n^{(2)}=0, \pm 1, \pm 2, K,
$$

where, $n^{(2)}$ represents the integer identifying fringes observed for shearing along the $x_{2}$ direction. Dividing Eq. (10) by $\omega$ gives

$$
\frac{S\left(x_{1}, x_{2}+\omega\right)-S\left(x_{1}, x_{2}\right)}{\omega}=\frac{n^{(2)} \lambda}{\omega}, \quad n^{(2)}=0, \pm 1, \pm 2, K,
$$

which for sufficiently small $\omega$ may be approximated by

$$
\frac{\partial S\left(x_{1}, x_{2}\right)}{\partial x_{2}}=\frac{n^{(2)} \lambda}{\omega}, \quad n^{(2)}=0, \pm 1, \pm 2, K .
$$

Using Eqs. (8) and (9) in Eq. (12), we have

$$
\frac{\partial S\left(x_{1}, x_{2}\right)}{\partial x_{2}}=\frac{n^{(2)} p}{\Delta}, \quad n^{(2)}=0, \pm 1, \pm 2, K .
$$

Generalizing the result to include wave-front shearing in either the $x_{1}$ or the $x_{2}$ direction, we have

$$
\frac{\partial S\left(x_{1}, x_{2}\right)}{\partial x_{\alpha}}=\frac{n^{(\alpha)} p}{\Delta}, \quad n^{(\alpha)}=0, \pm 1, \pm 2, K,
$$

where $n^{(\alpha)}$ represents the fringes observed for shearing along the $x_{\alpha}$ direction and $\alpha \in\{1,2\}$. Equations (14) are the governing equations for interferograms formed using the technique of CGS. A substantially more involved derivation of Eqs. (14) has been determined by using Fourier optics. ${ }^{21}$ However, the above simple demonstration of the physical principle of CGS suffices for the purposes of this article.

For a curved surface, the optical wave front may be interpreted in terms of the topography of the surface as follows. Consider a specularly reflective specimen whose curved surface (i.e., the reflector) can be expressed as 


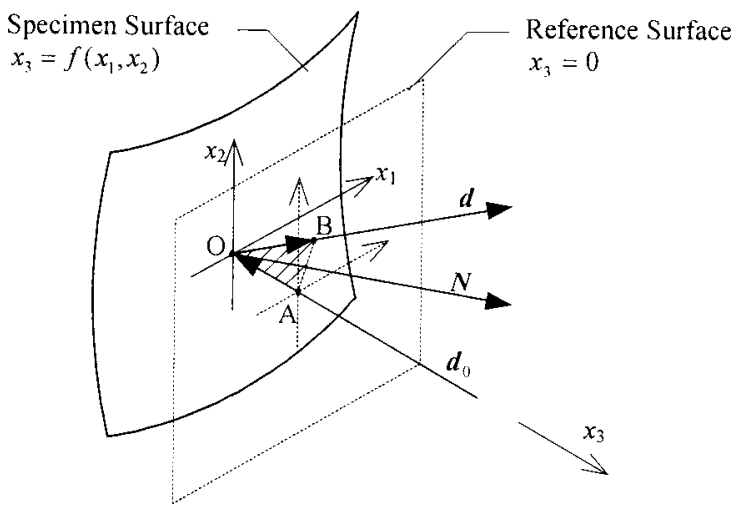

FIG. 8. Reflection of the incident wave front from the curved specimen surface.

$$
F\left(x_{1}, x_{2}, x_{3}\right)=x_{3}-f\left(x_{1}, x_{2}\right)=0 .
$$

The unit surface normal $N$ at a generic point $\left(x_{1}, x_{2}\right)$ of this curved surface is given by (see Fig. 8)

$$
\mathbf{N}=\frac{\nabla F}{|\nabla F|}=\frac{-f_{, 1} e_{1}-f_{, 2} e_{2}+e_{3}}{\sqrt{1+f_{, 1}^{2}+f_{, 2}^{2}}},
$$

where $f_{, \alpha}$ denotes in-plane gradient components of the specimen surface $x_{3}=f\left(x_{1}, x_{2}\right),(\alpha \in\{1,2\})$, and $e_{i}$ is the unit vector along the $x_{i}$ axis $(i=1,2,3)$. Now, consider an initially planar wave front incident on the specimen surface, such that the incident wave front is parallel to the $\left(x_{1}, x_{2}\right)$ plane. The unit incident wave propagation vector (vector normal to the incident wave front) is given as

$$
\mathbf{d}_{0}=-e_{3} .
$$

If the specimen surface was flat and occupied the $\left(x_{1}, x_{2}\right)$ plane, the unit reflected wave propagation vector (vector normal to the reflected wave front) would be collinear with the incident vector and would be given as

$$
\mathbf{d}=e_{3} .
$$

However, since the specimen surface is curved, the reflected wave front is perturbed, and the unit reflection propagation vector can be expressed as

$$
\mathbf{d}=\alpha e_{1}+\beta e_{2}+\gamma e_{3},
$$

where $\alpha\left(x_{1}, x_{2}\right), \beta\left(x_{1}, x_{2}\right)$, and $\gamma\left(x_{1}, x_{2}\right)$ denote the direction cosines of the reflected (perturbed) wave front. From the law of reflection the unit incident wave propagation vector $\mathbf{d}_{0}$, the unit reflected wave propagation vector $\mathbf{d}$, and the unit surface normal $\mathbf{N}$, are coplanar and related by (see Fig. 8)

$$
\mathbf{d} \cdot \mathbf{N}=\left(-\mathbf{d}_{0}\right) \cdot \mathbf{N}=e_{3} \cdot \mathbf{N} .
$$

This leads to the relation

$$
\mathbf{d}=\left(2 e_{3} \cdot \mathbf{N}\right) \mathbf{N}-e_{3} .
$$

Substituting Eq. (16) into Eq. (21) yields

$$
\mathbf{d}=\alpha e_{1}+\beta e_{2}+\gamma e_{3}=\frac{2\left(-f_{, 1} e_{1}-f_{, 2} e_{2}+e_{3}\right)}{1+f_{, 1}^{2}+f_{, 2}^{2}}-e_{3} .
$$

Thus,

$$
\alpha=\frac{-2 f_{, 1}}{1+f_{, 1}^{2}+f_{, 2}^{2}}, \quad \beta=\frac{-2 f_{, 2}}{1+f_{, 1}^{2}+f_{, 2}^{2}}, \quad \gamma=\frac{1-f_{, 1}^{2}-f_{, 2}^{2}}{1+f_{, 1}^{2}+f_{, 2}^{2}}
$$

To determine the change in the optical path length due to reflection from the curved specimen surface, as compared to reflection from a flat reference surface, consider the plane containing the unit incident and reflected vectors at any generic point $\left(x_{1}, x_{2}\right)$, as shown in Fig. 8. The net change in optical path length at point $\left(x_{1}, x_{2}\right)$ is given as

$$
\begin{aligned}
S\left(x_{1}, x_{2}\right) & =\left|\overline{O A}\left(x_{1}, x_{2}\right)\right|+\left|\overline{O B}\left(x_{1}, x_{2}\right)\right| \\
& =\left|\left(\frac{f\left(x_{1}, x_{2}\right)}{d\left(x_{1}, x_{2}\right) \cdot e_{3}}\right) d\left(x_{1}, x_{2}\right)\right|+\left|f\left(x_{1}, x_{2}\right) e_{3}\right| .
\end{aligned}
$$

Thus,

$$
S\left(x_{1}, x_{2}\right)=f\left(x_{1}, x_{2}\right)\left(\frac{2}{1-f_{, 1}^{2}-f_{, 2}^{2}}\right) .
$$

Assuming $\left|\nabla^{2} f\right| \ll 1$ and substituting Eq. (25) into Eq. (14), we get

$$
\frac{\partial f\left(x_{1}, x_{2}\right)}{\partial x_{\alpha}} \approx \frac{n^{(\alpha)} p}{2 \Delta}, \quad n^{(\alpha)}=0, \pm 1, \pm 2, K,
$$

where $\alpha \in\{1,2\}$. Equations (26) are the basic governing equations that relate CGS fringe contours to in-plane gradients of the specimen surface $x_{3}=f\left(x_{1}, x_{2}\right)$.

Now, in order to relate CGS interferograms of a given surface to its curvature, consider a curved specimen, as shown in Fig. 9(a). The normal at a point $P\left(\xi_{1}, \xi_{2}\right)$ on the surface is defined as

$$
\mathbf{a}_{3}=\frac{\mathbf{a}_{1} \times \mathbf{a}_{2}}{\left|\mathbf{a}_{1} \times \mathbf{a}_{2}\right|},
$$

where $\mathbf{a}_{1}$ and $\mathbf{a}_{2}$ are unit vectors tangent to the curvilinear coordinates axes $\left(\xi_{1}, \xi_{2}\right)$. Unit tangent vectors $\mathbf{a}_{1}$ and $\mathbf{a}_{2}$ are given in terms of position vector $\mathbf{r}\left(\xi_{1}, \xi_{2}, \xi_{3}\right)$ of the point $P\left(\xi_{1}, \xi_{2}\right)$ as $\mathbf{a}_{\alpha}=\partial r / \partial \xi_{\alpha}, \alpha \in\{1,2\}$.

The rate at which $\mathbf{a}_{3}$ varies between neighboring points provides a measure of curvature at the point of interest. Now,

$$
d \mathbf{a}_{3}=\frac{\partial \mathbf{a}_{3}}{\partial \xi_{\alpha}} d \xi_{\alpha}
$$

Note that $\partial \mathbf{a}_{3} / \partial \xi_{\alpha}$, are tangent vectors since $\mathbf{a}_{3} \cdot\left(\partial \mathbf{a}_{3} / \partial \xi_{\alpha}\right)$ $=0$. The curvature tensor $\boldsymbol{\kappa}$ is defined as the projections of the rate of change vectors $\partial \mathbf{a}_{3} / \partial \xi_{\alpha}$ along unit tangent vectors $\mathbf{a}_{1}$ and $\mathbf{a}_{2}$ as

$$
\boldsymbol{\kappa}_{\alpha \beta}=-\frac{\partial \mathbf{a}_{3}}{\partial \xi_{\alpha}} \cdot a_{\beta}, \quad \alpha, \beta \in\{1,2\} .
$$

Or, in terms of position vector $\mathbf{r}\left(\xi_{1}, \xi_{2}, \xi_{3}\right)$,

$$
\boldsymbol{\kappa}_{\alpha \beta}=\mathbf{a}_{3} \cdot \frac{\partial^{2} r}{\partial \xi_{\alpha} \partial \xi_{\beta}}, \quad \alpha, \beta \in\{1,2\} .
$$




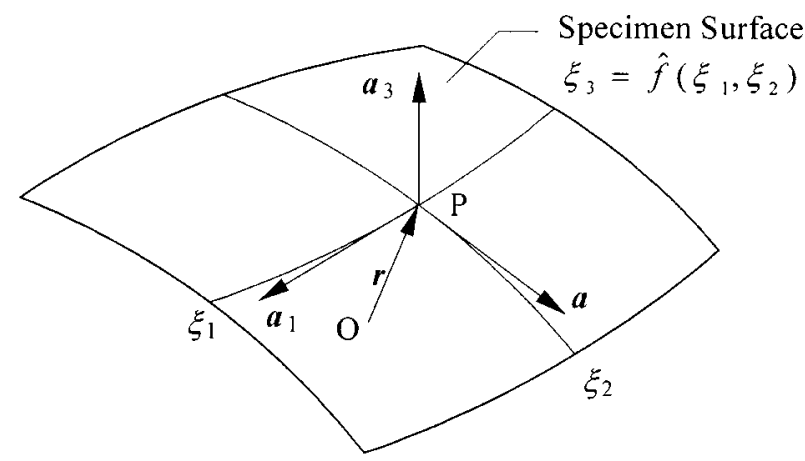

(a)

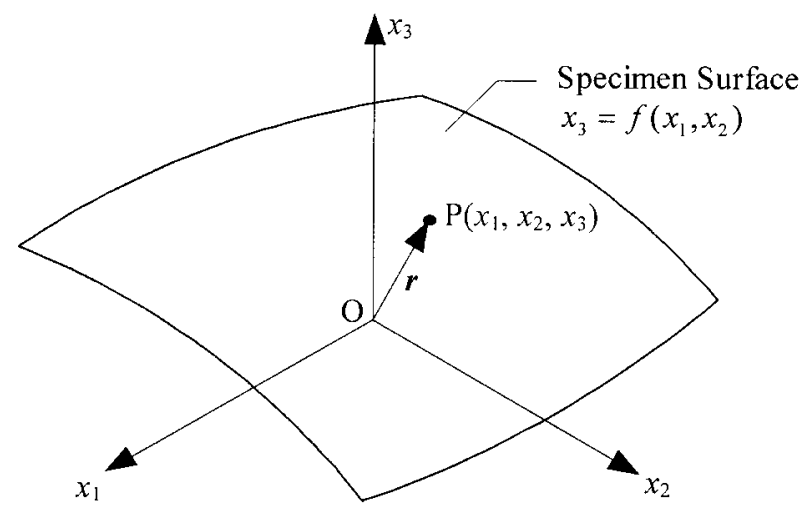

(b)

FIG. 9. (a) Curved specimen surface described in terms of curvilinear coordinates, and (b) shallow surface with small curvatures.

$\boldsymbol{\kappa}_{\alpha \beta}$ is the symmetric curvature tensor whose components $\boldsymbol{\kappa}_{11}$ and $\boldsymbol{\kappa}_{22}$ are termed as the "normal curvatures" and $\boldsymbol{\kappa}_{12}\left(=\boldsymbol{\kappa}_{21}\right)$ as the "twist." The principal values of $\boldsymbol{\kappa}_{\alpha \beta}$ are termed as the principal curvatures.

Consider the case of a shallow surface, $x_{3}=f\left(x_{1}, x_{2}\right)$, as shown in Fig. 9(b). The curvilinear coordinate systems reduce to

$$
x_{1}=\xi_{1}, \quad x_{2}=\xi_{2}, \quad x_{3}=\hat{f}\left(\xi_{1}, \xi_{2}\right)=f\left(x_{1}, x_{2}\right),
$$

and

$$
\mathbf{r}\left(x_{1}, x_{2}, x_{3}\right)=x_{1} \mathbf{e}_{1}+x_{2} \mathbf{e}_{2}+f\left(x_{1}, x_{2}\right) \mathbf{e}_{3} .
$$

Thus,

$$
\boldsymbol{\kappa}_{\alpha \beta}=\mathbf{a}_{3} \cdot \frac{\partial^{2} \mathbf{r}}{\partial x_{\alpha} \partial x_{\beta}}=\frac{f_{, \alpha \beta}}{\sqrt{1+f_{, 1}^{2}+f_{, 2}^{2}}}, \quad \alpha, \beta \in\{1,2\} .
$$

For small curvatures, $\left|\nabla^{2} f\right| \ll 1$, and thus

$$
\boldsymbol{\kappa}_{\alpha \beta} \approx f_{, \alpha \beta}, \quad \alpha, \beta \in\{1,2\} .
$$

Substituting Eq. (26) into Eq. (34), we get the basic equations that relate CGS fringes to specimen curvature,

$$
\begin{aligned}
& \boldsymbol{\kappa}_{\alpha \beta}\left(x_{1}, x_{2}\right) \approx \frac{\partial^{2} f\left(x_{1}, x_{2}\right)}{\partial x_{\alpha} \partial x_{\beta}} \approx \frac{p}{2 \Delta}\left(\frac{\partial n^{(\alpha)}\left(x_{1}, x_{2}\right)}{\partial x_{\beta}}\right), \\
& n^{(\alpha)}=0, \pm 1, \pm 2, K,
\end{aligned}
$$

where, $\alpha \in\{1,2\}$. Equation (35) is the principal governing equation for determining curvature tensor fields $\boldsymbol{\kappa}_{\alpha \beta}\left(x_{1}, x_{2}\right)$, $(\alpha, \beta \in\{1,2\})$, from CGS interferograms. In this manner, CGS interferograms provide a full-field technique for determining the instantaneous value of the specimen curvature tensor at any point $\left(x_{1}, x_{2}\right)$.

\section{CGS FRINGE ANALYSIS USING THE FOURIER TRANSFORM}

In this section, we present the methodology used in automatically postprocessing the CGS fringe patterns captured in this work by a high-resolution charge-coupled-device (CCD) camera. Our goal is to take advantage of the obtained, high-resolution, digital information and to increase our data accuracy. To achieve this, a Fourier transform phase measurement technique ${ }^{22}$ is used to extract the full-field phaseangle information from the CGS images and to differentiate it in order to obtain the desired curvature fields. The interference fringe patterns are captured in real time by the CCD camera and the video signal is subsequently forwarded to a PC-based digital processing system. The principles involved in this procedure are described below.

In general, the intensity field of the CGS interference fringe patterns can be expressed by

$$
I\left(x_{1}, x_{2}\right)=a\left(x_{1}, x_{2}\right)+b\left(x_{1}, x_{2}\right) \cos \delta\left(x_{1}, x_{2}\right),
$$

where $I\left(x_{1}, x_{2}\right)$ is the intensity of the fringe pattern at a field point $\left(x_{1}, x_{2}\right)$ on the specimen surface, $a\left(x_{1}, x_{2}\right)$ and $b\left(x_{1}, x_{2}\right)$ are its background intensity level and the fringe visibility, respectively, and $\delta\left(x_{1}, x_{2}\right)$ is the phase-angle term contributed by the deformation of the specimen. This intensity distribution can also be expressed in terms of a sum of complex field quantities as follows:

$$
I\left(x_{1}, x_{2}\right)=a\left(x_{1}, x_{2}\right)+c\left(x_{1}, x_{2}\right)+c *\left(x_{1}, x_{2}\right),
$$

where $c\left(x_{1}, x_{2}\right)=\frac{1}{2} b\left(x_{1}, x_{2}\right) e^{i \delta\left(x_{11}, x_{2}\right)}$, and $c^{*}\left(x_{1}, x_{2}\right)$ is its complex conjugate.

By taking the Fourier transform of this intensity distribution, we get

$$
I\left(\omega_{1}, \omega_{2}\right)=A\left(\omega_{1}, \omega_{2}\right)+C\left(\omega_{1}, \omega_{2}\right)+C^{*}\left(-\omega_{1},-\omega_{2}\right),
$$

with $\omega_{1}$ and $\omega_{2}$ being the spatial frequencies in the transform domain, and $C^{*}\left(-\omega_{1},-\omega_{2}\right)$ the conjugate symmetric of $C\left(\omega_{1}, \omega_{2}\right) . A\left(\omega_{1}, \omega_{2}\right)$ contains the constant- and lowfrequency information due to slow variations of the intensity background. $C\left(\omega_{1}, \omega_{2}\right)$ and $C^{*}\left(-\omega_{1},-\omega_{2}\right)$ carry basically the same information. By using bandpass filtering in the spatial frequency domain, $A\left(\omega_{1}, \omega_{2}\right)$ and either of $C\left(\omega_{1}, \omega_{2}\right)$ or $C^{*}\left(-\omega_{1},-\omega_{2}\right)$ are filtered out. The remaining spectrum $C\left(\omega_{1}, \omega_{2}\right)$ or $C^{*}\left(-\omega_{1},-\omega_{2}\right)$ contains the required phaseangle information $\delta\left(x_{1}, x_{2}\right)$, so that its inverse Fourier trans- 


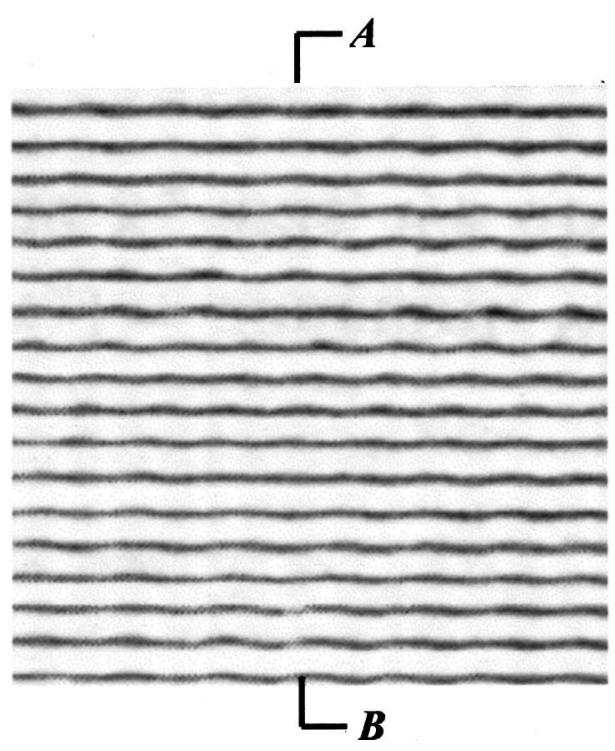

(a)

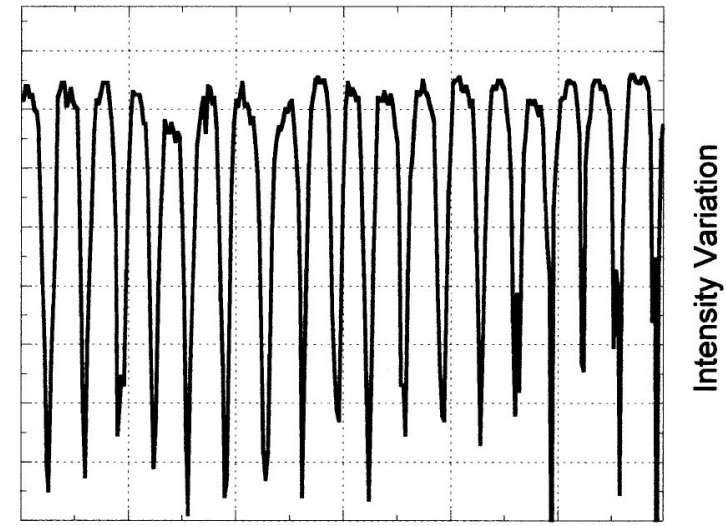

$x_{2}$

(b)

FIG. 10. CGS fringe pattern of spherical wave front $(p=25 \mu \mathrm{m}$ and $\Delta$ $=22 \mathrm{~mm}$ ). (a) CGS fringe patterns, and (b) intensity profile along $A-B$ in (a).

form gives us back either complex $c\left(x_{1}, x_{2}\right)$ or $c^{*}\left(x_{1}, x_{2}\right)$, respectively. The phase distribution $\delta\left(x_{1}, x_{2}\right)$ is then obtained as follows:

$$
\delta\left(x_{1}, x_{2}\right)=\tan ^{-1} \frac{\operatorname{Im}\left[c\left(x_{1}, x_{2}\right)\right]}{\operatorname{Re}\left[c\left(x_{1}, x_{2}\right)\right]},
$$

where $\operatorname{Re}[$ ] and $\operatorname{Im}[$ ] represent real and imaginary parts, respectively.

To verify the above Fourier transform fringe methodology, a well-defined spherical wave front was examined, as shown in Fig. 10(a). This spherical wave front is generated by passing a collimated laser beam through a planoconvex lens of focal length $f_{l}(=546 \mathrm{~mm})$, and a known, constant radius of curvature $2 f_{l}$. We will now apply the Fourier transform technique to recover this curvature accurately. The spherical wave front passing through the planoconvex lens can be described by

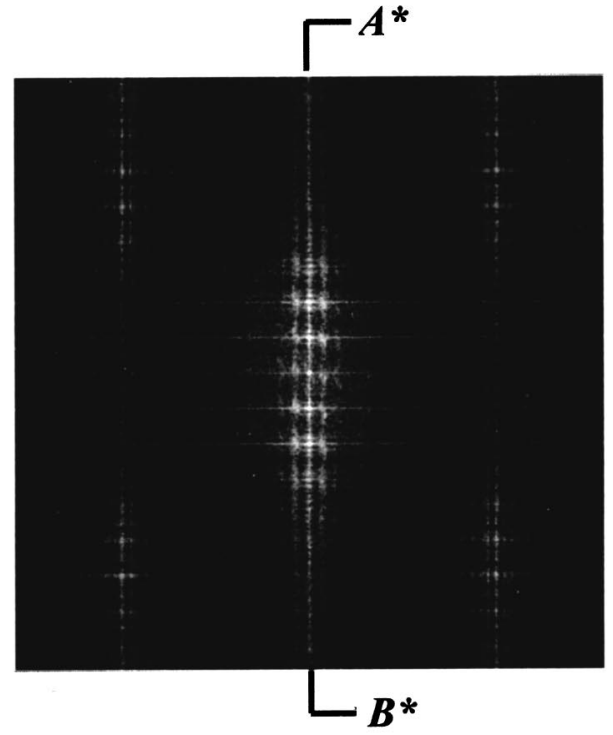

(a)

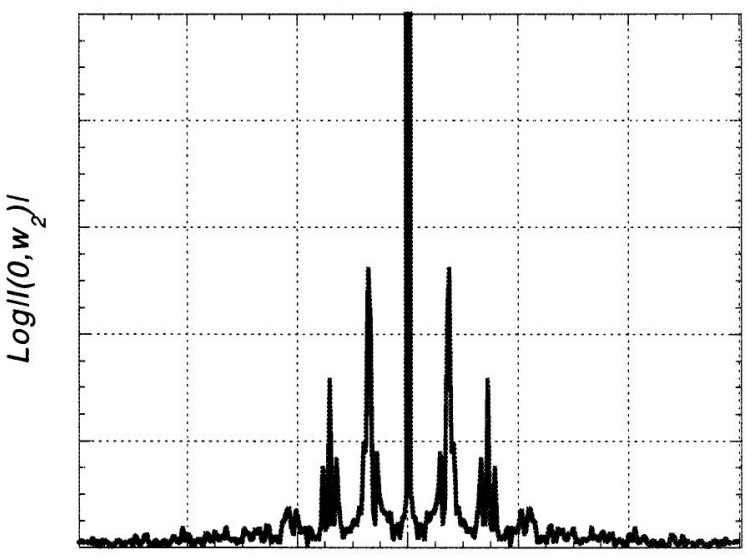

$x_{2}$

(b)

FIG. 11. Two-dimensional Fourier transform of spherical wave front shown in Fig. 10. (a) Fourier transform magnitude in the frequency domain $\left(\omega_{1}, \omega_{2}\right)$, and (b) magnitude variation along $A^{*}-B^{*}$ in (a).

$$
S\left(x_{1}, x_{2}\right)=\frac{x_{1}^{2}+x_{2}^{2}}{2 f_{l}} .
$$

When grating lines are oriented such that their principal direction is parallel to the $x_{2}$ axis, CGS provides vertical gradient component information in the form of parallel fringes, whose equation is given by

$$
\frac{\partial S}{\partial x_{2}}=\frac{x_{2}}{f_{l}}=\frac{n^{(2)} p}{\Delta} .
$$

Figure 10(a) shows the raw digitally recorded CGS images. The noisy intensity variation corresponding to a vertical cross section $A B$ is displayed in Fig. 10(b). The Fourier transform is then applied, and its two-dimensional power spectrum is shown here in Fig. 11(a), and the corresponding cross section in Fourier space $\left(A^{*} B^{*}\right)$ is displayed in Fig. 11(b). By applying the procedure outlined before, through 


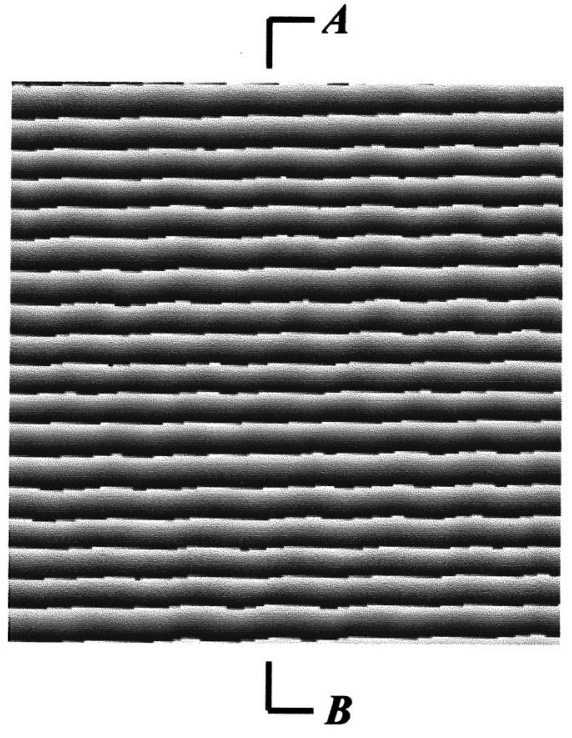

(a)

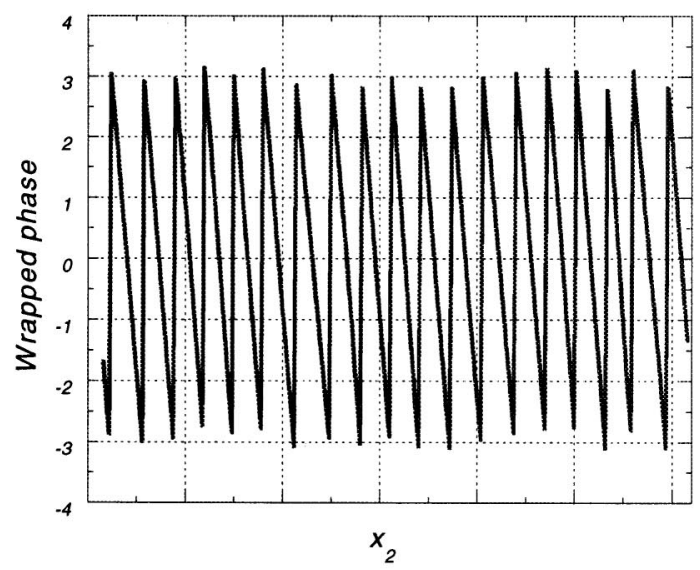

(b)

FIG. 12. Wrapped phase information by inverse Fourier transform using bandpass filtering: (a) wrapped phase variation, and (b) wrapped phase variation along $A-B$ in (a).

bandpass filtering, we invert the transform and display its two-dimensional "wrapped" phase-angle field $\delta\left(x_{1}, x_{2}\right)$ in Fig. 12(a) and the corresponding cross-sectional information in Fig. 12(b). In Fig. 12, the phase angle is wrapped or restricted to vary between the values of $-\pi$ and $\pi$. It should be noted that the filtering process has eliminated the low- and high-frequency noise, resulting in information which is relatively "crisp" compared to that displayed in Figs. 10(a) and 10(b).

The results of Fig. 12 are then "unwrapped" by extrapolating between the discrete peaks and assigning a continuous spectrum of fringe numbers, thus creating the smooth contours of phase information and fringe order shown in Figs. 13(a) and 13(b), respectively. The fringe order represents the linear first partial derivative of $S\left(x_{1}, x_{2}\right)$ with respect to $x_{2}$. The curvature is then obtained by numerically differentiating this variation once more with respect to $x_{2}$.

A three-dimensional representation of the entire curva-

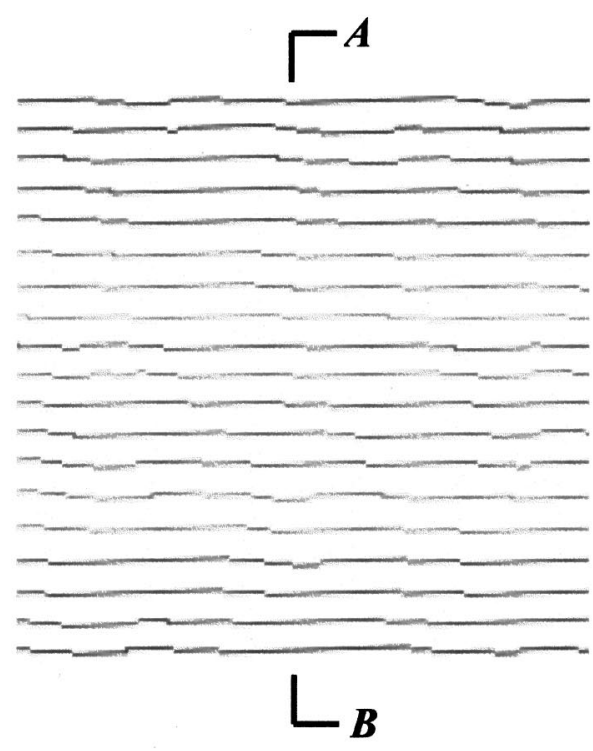

(a)

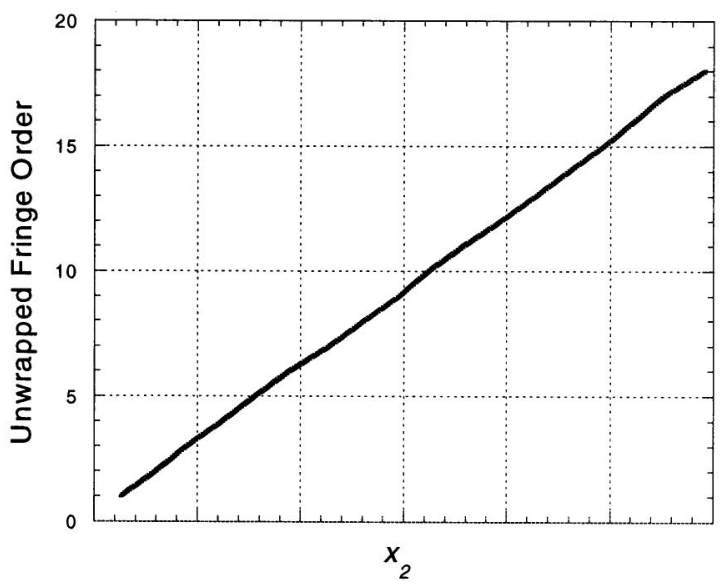

(b)

FIG. 13. Unwrapped phase information: (a) unwrapped phase variation, the lines indicate the same fringe order contours; and (b) fringe order variation $A-B$ in (a).

ture field $\kappa_{22}\left(x_{1}, x_{2}\right)$ obtained by this method is shown here is Fig. 14. Notwithstanding small fluctuations that result from the differentiation procedure, the level of curvature is constant and equal to the known curvature of the lens $\kappa_{22}$ $=\left(2 f_{l}\right)^{-1} \approx 1092 \mathrm{~mm}$.

\section{APPLICATION OF CGS FOR CURVATURE MEASUREMENT OF A FILM-SUBSTRATE SYSTEM}

The technique of CGS described in the previous sections was applied to determine instantaneous full-field maps of curvature of a film-substrate system. The behavior of a system consisting of a thin Al film of thickness $h_{f}=6 \mu \mathrm{m}$ deposited on a circular $\mathrm{Si}$ wafer of radius $R=25.4 \mathrm{~mm}$ and nominal thickness of $h_{s}=105 \mu \mathrm{m}$ was studied in the laboratory. The wafer was a single crystal with a (100) normal orientation and the film had a fine-grained columnar polycrystalline microstructure. The wafer was flat prior to film deposition at a temperature of $87.5^{\circ} \mathrm{C}$, where, after, it was 


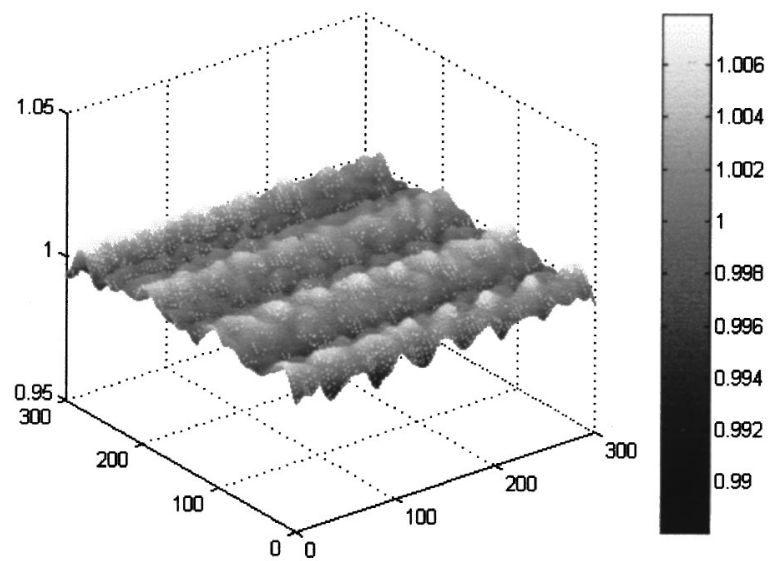

FIG. 14. Normalized curvature variation $\kappa_{22} / \kappa_{\text {lens }}$ by using $\kappa_{22}\left(x_{1}, x_{2}\right)$ $\approx p / 2 \Delta\left(\partial n^{(2)}\left(x_{1}, x_{2}\right) / \partial x_{2}\right)$.

cooled to room temperature. The temperature was then cycled between room temperature and the film deposition temperature of $87.5^{\circ} \mathrm{C}$. This was done in a temperature control chamber over approximately a $2 \mathrm{~h}$ time cycle. It was assumed that within this range the deformation remained elastic.

During thermal cycling, the deformation of the sample was remotely monitored through a quartz window attached to the heating chamber. The measurement was performed by means of the optical coherent gradient sensing method and a CCD camera which continuously recorded the CGS fringes. The CGS method is noninvasive, allows for continuous observation over the full field of view. Standard interferometric methods are sensitive to rigid-body motion of the sample; and displacement data thus obtained must be differentiated twice numerically to obtain curvature fields. The CGS method, on the other hand, permits a direct measure of surface gradients; it is, therefore, insensitive to rigid-body motions and in addition only one differentiation of data is required to extract the curvature fields. The technique is capable of resolving radii of curvature up to approximately $10 \mathrm{~km}^{17}$

In Fig. 15, a set of CGS fringe patterns captured by the $\mathrm{CCD}$ camera at the $\mathrm{Al}$ film deposition temperature of $87.5^{\circ} \mathrm{C}$ are shown. The bright fringe lines are level curves of slope in the vertical $x_{2}$ direction of each figure, while the spacing of the lines is an indicator of curvature of material lines in the vertical direction. Each image corresponds to different sample rotations by increments of $\pi / 4$. Figures 15 (a), 15(b), 15(c), and 15(d) reveal the large spacing of fringes in the central portion of the sample. This implies that the Al film thickness is rather uniform there since the Si substrate was flat before deposition.

However, high fringe density near the edge clearly represents nonuniform film thickness in a boundary layer of approximately $6 \mathrm{~mm}$. From a simple profile calculation, using fringe information in this area, a simple parabolic equation was found to provide a good fit of the nonuniform thickness profile, which is shown schematically here in Fig. 16. This higher fringe density area starts at $r>0.75 R$, and the
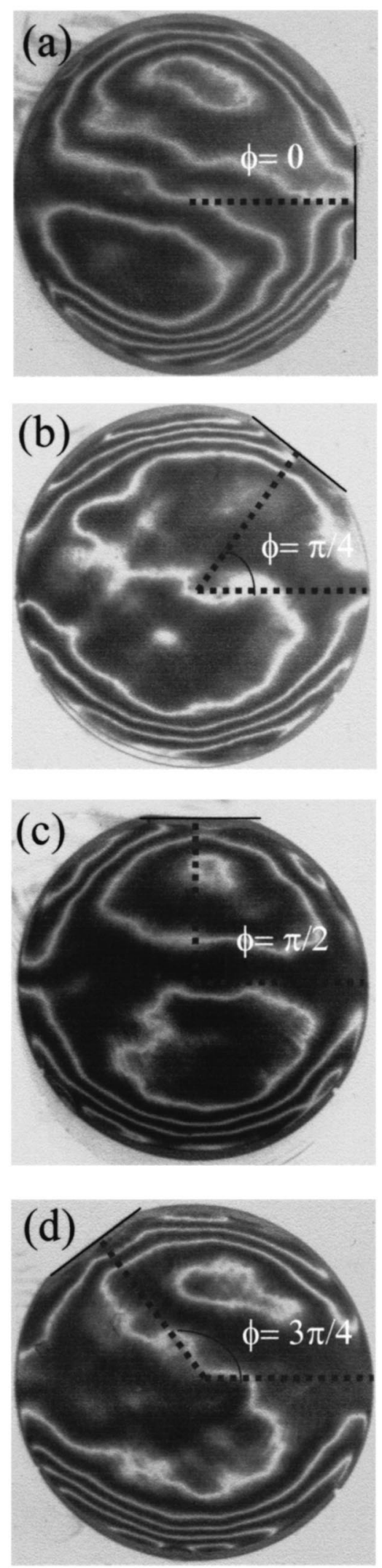

FIG. 15. Set of CGS fringe patterns for the $\mathrm{Al}$ film deposited on the $\mathrm{Si}$ substrate at deposition temperature $87.5^{\circ} \mathrm{C}$. Images (a), (b), (c), and (d) correspond to different sample orientations $(\phi=0, \pi / 4, \pi / 2$, and $3 \pi / 4$, respectively). 


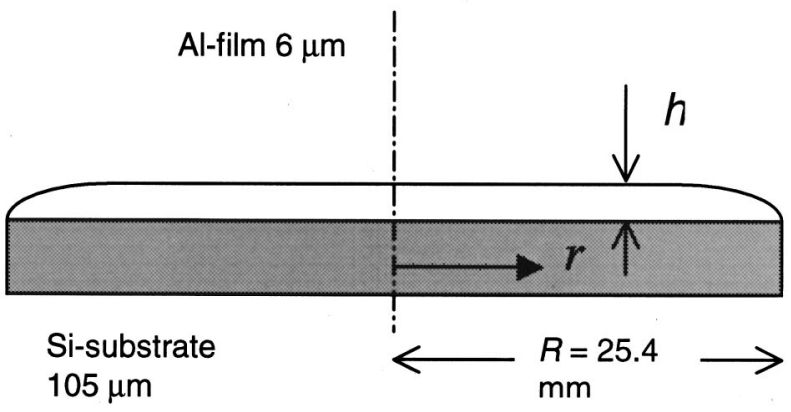

FIG. 16. Al-film thickness profile; $h_{f}=6 \mu \mathrm{m}$ for $r \leqslant 0.75 R, h$ $=h_{f} \sqrt{1-(r-3 R / 4)^{2} /(R / 4)^{2}}$ for $r>0.75 R$.

film thickness profile then is approximated by a parabolic equation, as shown in Fig. 16.

After recording the thickness profile, the system was gradually cooled from deposition temperature down to room temperature. A sequence of CGS interferograms correspond- ing to this cooling process are shown in Fig. 17. Images (a) and (c) correspond to temperature changes of $7.5^{\circ} \mathrm{C}$ while images (b) and (d) correspond to temperature changes of $52.5^{\circ} \mathrm{C}$. Images (a), (b) and (c), (d) correspond to two different sample orientations ( $\phi=\pi / 4$ and $3 \pi / 4$, respectively). In images (a) and (c), the CGS fringes are similar and are horizontal in the center. The similarity between (a) and (c) indicates that at that temperature the wafer, although not spherical, remains axially symmetric everywhere. In images (b) and (d), the curvature has become quite large, as evidenced by the close spacing of the fringes. It is clear that the sample has taken on a curved shape, which is neither axisymmetric nor spatially uniform, a situation which is clearly deduced by the pronounced nonuniformity of the fringes of Fig. 17(d). Magnified views of the central region of samples (b) and (d) of Fig. 17 are shown in Fig. 18. It should be noted that the biggest fringe density (highest curvature) and the lowest fringe density (lowest curvature) are observed in
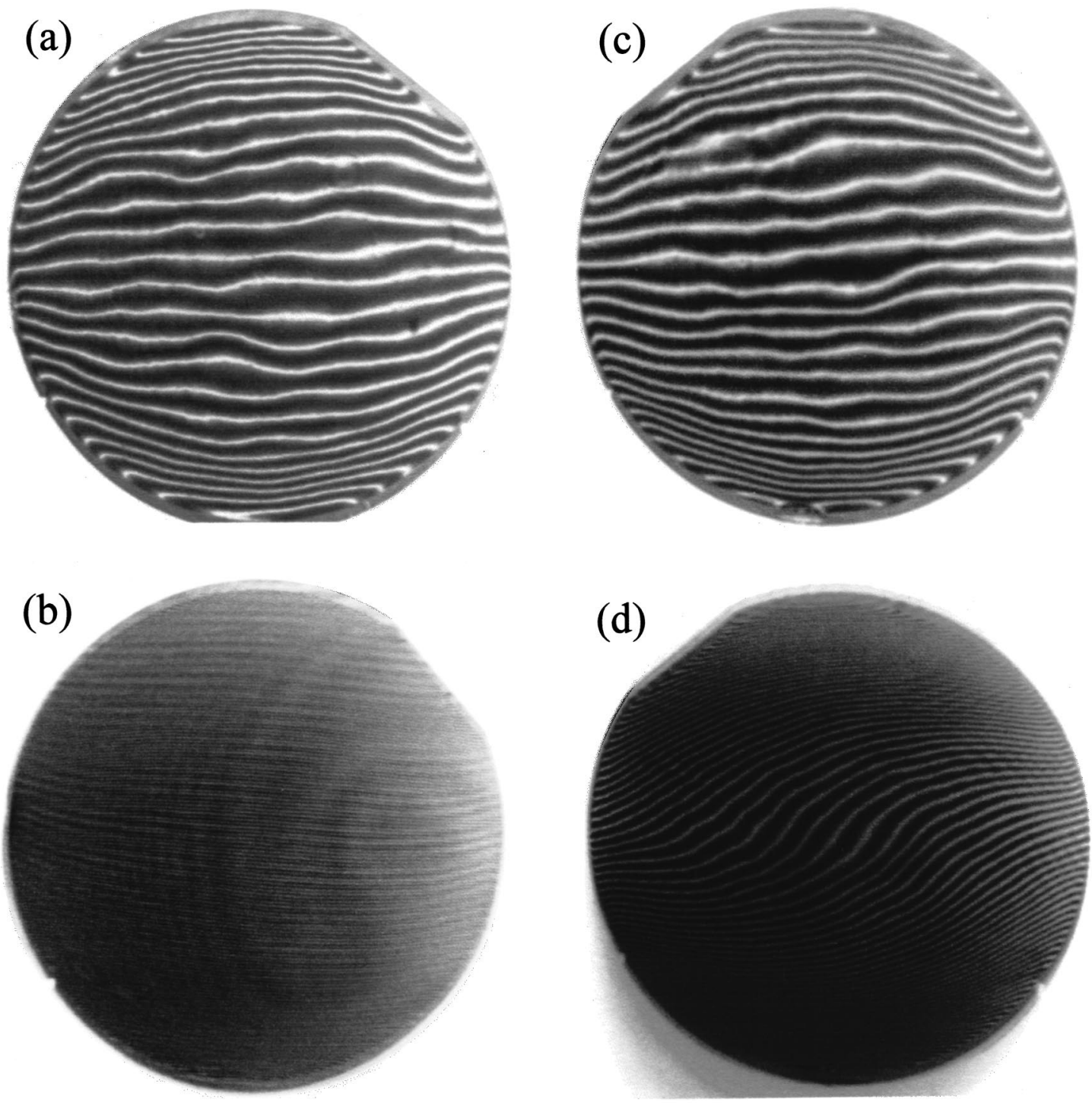

FIG. 17. Series of CGS fringe patterns for an $\mathrm{Al}$ film deposited on a thin Si substrate subjected to temperature changes of $7.5^{\circ} \mathrm{C}[(\mathrm{a})$ and $(\mathrm{c})]$ and $52.5^{\circ} \mathrm{C}[(\mathrm{b})$ and (d)], respectively. Different sample orientations, $(\phi=\pi / 4[(\mathrm{a}),(\mathrm{b})]$ and $3 \pi / 4[(\mathrm{c}),(\mathrm{d})])$, are shown. 


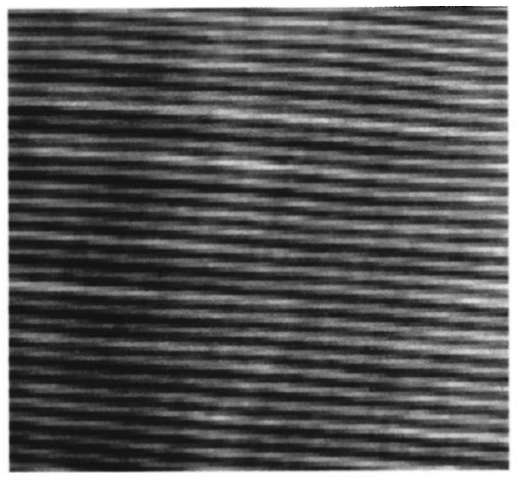

(a)

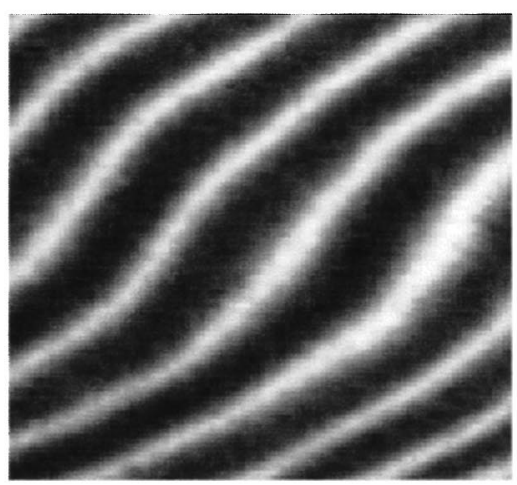

(b)

FIG. 18. Magnified CGS fringe patterns near the center portion of the sample at temperature changes of $52.5^{\circ} \mathrm{C}$ [see Figs. 17(b) and 17(d), respectively].

fringe patterns corresponding to the two orthogonal directions $\phi=\pi / 4$ and $\phi=3 \pi / 4$, respectively.

These seem to be principal directions of curvature. For a case in which the deformations are large but the deformed shape is still axisymmetric, similar to the situation illustrated in images (a) and (c) of Fig. 17, the distribution of curvature along four radial surface lines at angles of $\pi / 4$ to each other was extracted from the data. The results are displayed in Fig. 19 in the form of radial curvature $K_{r}$ vs $r / R$ along these radial lines. Figure 19 clearly shows that the curvature, although largely still axisymmetric, is clearly nonuniform, increasing steadily from the center of the wafer to the outer edge. The solid line in Fig. 19 is the curvature distribution predicted from a finite-element simulation of the sample under the conditions of the experiment. This calculation takes into consideration the nonuniform thickness of the film as obtained by the experimental measurements and as approximated in Fig. 16. The general features of this radial variation are described in the earlier section of this article.

It is clear from Fig. 19 that the numerics captures well the experimentally measured curvature variation. Throughout most of the specimen center $r<0.75 R$ does a good job in predicting the curvature right at the specimen rim but underestimates the maximum curvature at $r \sim 0.8 R$ by approximately $15 \%$. This discrepancy is most probably related to the fact that the calculation does not properly take into account

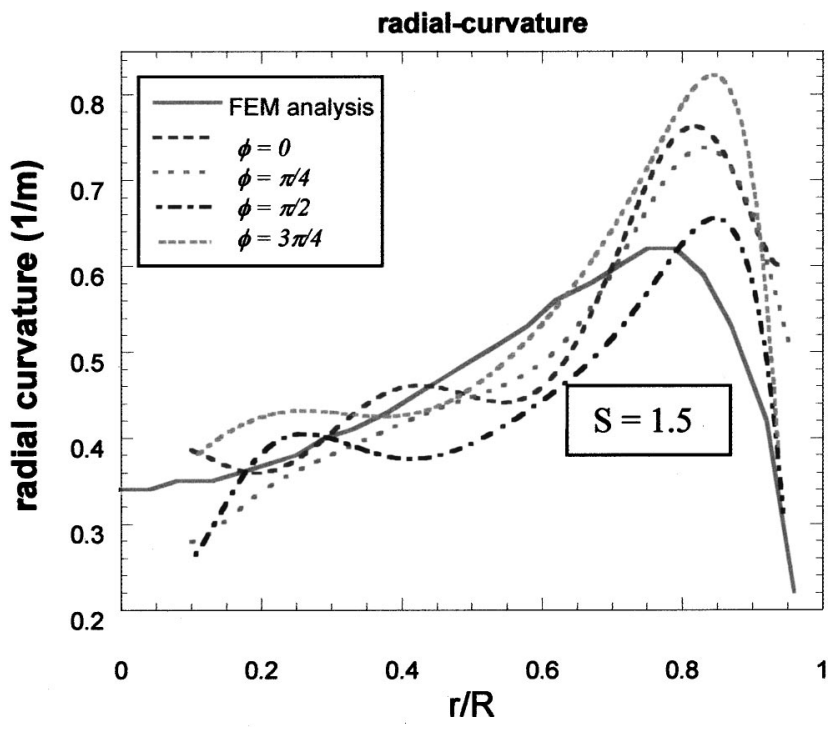

FIG. 19. Curvature of a radial line vs distance along four radial lines when the deformation is still axisymmetric but beyond the linear range. The experimental data points are from separate lines at $\pi / 4$ to each other. The solid line is from a finite-element simulation of the sample $\left(\Delta T=22.5^{\circ} \mathrm{C}\right)$.

natural specimen imperfections, and the substrate anisotropy. However, both experiment and numerics clearly exibit the physical picture discussed in the theoretical part of this article. Indeed, for a temperature change at $\Delta T \sim 22.5^{\circ} \mathrm{C}$ [corresponding to the value of normalized mismatch strain $S$ of 1.5, where $\left.\varepsilon_{m}=\left(\alpha_{\mathrm{Al}}-\alpha_{\mathrm{Si}}\right) \Delta T\right]$, the deformation is still largely radially symmetric as discussed earlier (see Fig. 5). However, the specimen is rather flat in the center, becoming increasingly curved towards the edges.

We now turn to investigating the bifurcation behavior discussed in Sec. III. We concentrate our attention on a small area of the sample near its center and monitor the curvature there as a function of thermally induced mismatch strain resulting from cooling the sample from its deposition temperature to room temperature. Figure 20 shows the observed and calculated curvatures near $r=0$ as functions of the normalized mismatch strain $S$ as the temperature change becomes large enough to drive the behavior of the wafer into the postbifurcation regime. Again, the results are presented in the nondimensional form, which is consistent among all results reported here. The two dashed curves are measured curvature of lines in the directions of largest curvature $(\phi=\pi / 4)$ and smallest curvature $(\phi=3 \pi / 4)$ on the asymmetric deformation branch. It was anticipated in Fig. 5 of Sec. III that the bifurcation point would be largely obscured by even very small imperfections in the system, and this seems to be the case here. Nonetheless, it appears that the deformation is no longer axisymmetric when $S$ has increased beyond 1.5, whereas it appears to be reasonably axisymmetric up to this point. As before, the exact sample geometry tested in the laboratory was simulated by means of the finite-element calculation, and the solid curves in Fig. 20 represent the result. The predicted mismatch strain level for bifurcation is approximately $S=1.4$. The film and substrate moduli differ in this case, so this system does not fall strictly within the class represented by Fig. 5 in Sec. III. The curvature as obtained 


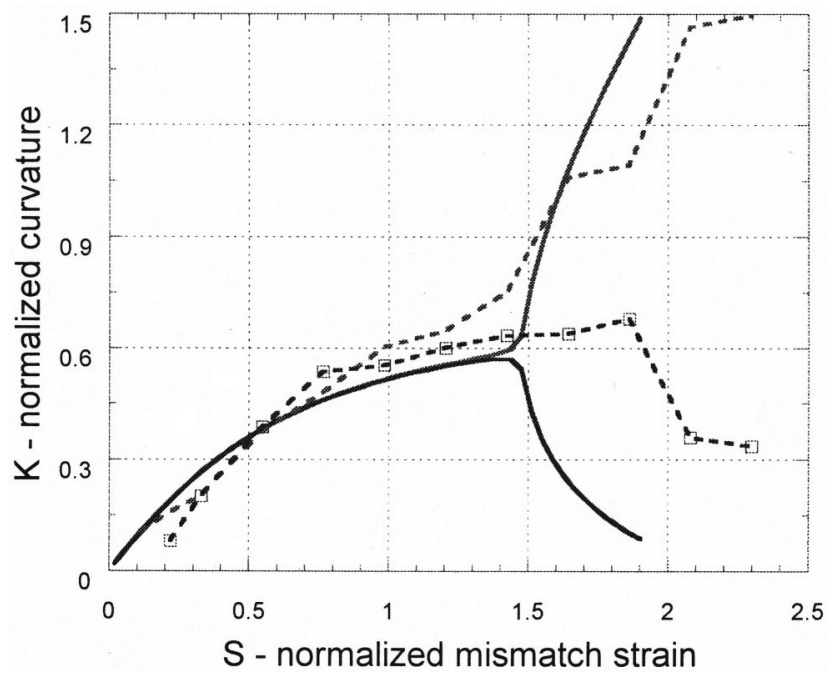

FIG. 20. Radial curvature near the center of the sample as mismatch strain is increased through the bifurcation point. The radial lines identified as $\phi$ $=\pi / 4$ and $3 \pi / 4$ are those which undergo maximum and minimum curvature, respectively, in the postbifurcation range. The dashed lines are from the experiment and the solid lines with matching symbols are from a finiteelement simulation of the sample.

from the simulation is subject to a small uncertainty because it is obtained by differentiating nodal displacement data. Given the anticipated strong effect of natural imperfections on the bifurcation point, the agreement between the experimentally observed mismatch strain for bifurcation and the equivalent theoretically predicted value is surprisingly good. Beyond this mismatch strain the results remain qualitatively very similar, clearly showing the tendency of the sample to assume a more cylindrical shape (curvature along one direction increasing many times more than its orthogonal counterpart).

\section{ACKNOWLEDGMENTS}

The authors would like to acknowledge financial support through the Jet Propulsion Laboratory Center for Integrated Space Microsystems, System on a Chip (SOAC) program. Many helpful discussions with Dr. E. Kolawa of JPL are also acknowledged.

${ }^{1}$ P. A. Flinn, Mater. Res. Soc. Symp. Proc. 130, 41 (1989).

${ }^{2}$ W. D. Nix, Metall. Trans. A 20A, 2217 (1989).

${ }^{3}$ J. A. Floro, E. Chason, S. R. Lee, R. D. Twesten, R. Q. Hwang, and L. B. Freund, J. Electron. Mater. 26, 969 (1997).

${ }^{4}$ J. A. Floro, E. Chason, R. D. Twesten, R. Q. Hwang, and L. B. Freund, Phys. Rev. Lett. 79, 3946 (1997).

${ }^{5}$ J. A. Floro, G. A. Lucadamo, E. Chason, L. B. Freund, M. Sinclair, R. D. Twesten, and R. Q. Hwang, Phys. Rev. Lett. 80, 4717 (1998).

${ }^{6}$ G. G. Stoney, Proc. R. Soc. London, Ser. A 82, 172 (1909).

${ }^{7}$ L. B. Freund, J. Cryst. Growth 132, 341 (1993).

${ }^{8}$ L. B. Freund, J. Mech. Phys. Solids 44, 723 (1996).

${ }^{9}$ F. Kroupa, Z. Knesi, and J. Valach, Acta Tech. CSAV 38, 29 (1993).

${ }^{10}$ M. Finot and S. Suresh, J. Mech. Phys. Solids 44, 683 (1996).

${ }^{11}$ K. O. Friedrichs, and J. J. Stoker, Proc. Natl. Acad. Sci. U.S.A. 25, 535 (1939).

${ }^{12}$ K. O. Friedrichs and J. J. Stoker, J. Appl. Mech. 9, A7 (1942).

${ }^{13}$ W. H. Wittrick, Quarterly J. Appl. Math. 6, 15 (1953).

${ }^{14}$ M. W. Hyer, J. Compos. Mater. 16, 318 (1982).

${ }^{15}$ L. B. Freund, J. Mech. Phys. Solids 48, 1159 (2000).

${ }^{16}$ M. Finot, I. A. Blech, S. Suresh, and H. Fujimoto, J. Appl. Phys. 81, 3457 (1997).

${ }^{17}$ A. J. Rosakis, R. P. Singh, Y. Tsuji, E. Kolawa, and N. R. Moore, Thin Solid Films 325, 42 (1998).

${ }^{18}$ C. B. Masters and N. J. Salamon, Int. J. Eng. Sci. 31, 915 (1993).

${ }^{19}$ N. J. Salamon and C. B. Masters, Int. J. Solids Struct. 32, 473 (1995).

${ }^{20}$ L. B. Freund, Mater. Res. Soc. Symp. Proc. 436, 393 (1997).

${ }^{21}$ Y. J. Lee, J. Lambros, and A. J. Rosakis, Opt. Lasers Eng. 25, 25 (1996).

${ }^{22}$ K. Creath, Prog. Opt. 26, 350 (1988). 